\title{
Satisfação dos usuários da rede de Atenção Primária de Porto Alegre
}

\section{Users' satisfaction with Porto Alegre's Primary Care Network.}

\section{Resumo}

Aline do Amaral Zils

Rodrigo Caprio Leite de Castro² Mônica Maria Celestina de Oliveira²

Erno Harzheim²

Bruce Bartholow Duncan²

A satisfação dos usuários é um importante desfecho para avaliar serviços de saúde. Este estudo trata-se de uma avaliação do grau de satisfação dos usuários em relaçào à última consulta realizada na rede de atenção primária à saúde de Porto Alegre-RS, tendo como objetivo comparar a satisfação de usuários que avaliam a qualidade do processo de atenção recebida como sendo de alto escore de APS com a de usuários que a avaliam como baixo escore de APS. Estudo transversal de base populacional, utilizando o Instrumento de Avaliação da Atenção Primária, Primary Care Assesment Tool - PCATool, que possibilita a classificação dos serviços de saúde no seu grau de orientação à APS por meio do escore geral de atenção primária, definindo-o como alto ou baixo, de acordo com a experiência dos usuários. A satisfação do usuário foi medida por um questionário composto de doze questões referentes à última consulta, sendo medida por uma escala Likert de 5 itens: "muito bom", "bom", "regular", "ruim" e "muito ruim". Para a análise, os resultados foram agrupados em duas classificações, sendo "satisfeito", quando referido "muito bom" ou "bom", e "insatisfeito" quando referido algum dos demais itens. Observou-se diferença significativa nas 12 variáveis que refletem a satisfação em vários aspectos da consulta, demonstrando maior satisfação em usuários que classificaram o seu serviço como de alto escore. A avaliação geral da consulta apresentou $95,6 \%$ e $73,5 \%$ de "satisfeitos" nos serviços com alto e baixo escore de APS, respectivamente $(p<0,001)$. Usuários de serviços com alto grau de orientação à atenção primária obtêm maior satisfação em suas consultas. Essa maior satisfação pode beneficiar o processo decisório do paciente frente às recomendações médicas realizadas.

Palavras-chave: Atenção Primária à Saúde;

Avaliação de Serviços de Saúde; Estudos

Transversais.
Key Words: Primary Health Care; Evaluation

of Health Services; Cross-Sectional Studies.

'Fscola de Enfermagem, Universidade Federal do Rio Grande do Sul, Porto Alegre, Rio Grande do Sul, Brasil.

2Programa de Pós-Graduação em Epidemiologia, Faculdade de Medicina, Universidade Federal do Rio Grande do Sul, Porto Alegre, Rio Grande do Sul, Brasil. 


\section{Abstract}

Users' satisfaction is an important end-point to evaluate bealth services. This study bas to do with an assessment of the level of user's satisfaction with relation to the last visit beld in the primary bealth care network of Porto Alegre - RS, whose objective is to compare satisfaction of the users that assess the quality of the care process received as being of bigh APS score with that of the users who evaluate it as low APS score. A crass-sectional study based on the population, using the Primary Care Assessment Tool - PCATool, which enables the classification of the bealth services at the level of gridance to APS by means of the general score of primary care, defining it as bigh or low, in accordance nith the users' experience. The satisfaction of the user mas measured by a questionnaire consisting of thelve questions relative to the last visit, being measured by a 5-item Likert scale: "very good", "good", "regular", "bad" and "very bad". For the analysis, the results were grouped into two classifications, i.e. "satisfied", when referred as "very good" or "good", and "dissatisfied" when referred to any of the other items. A significant difference was noticed in the 12 variables reflecting satisfaction in several aspects of the visit, showing bigher satisfaction in users that classiffed the service as being of bigh score. The general evaluation of the service presented $95.6 \%$ and $73.5 \%$ of "satisfied" in the services with bigh and low APS score, respectively $(p<0.001)$. Users of services with bigh level of guidance to primary care obtain bigher satisfaction in their visits. This higber satisfaction can benefit the decision-making process of the patient in face of the medical recommendations made.

\section{Introdução}

A satisfação dos usuários é um importante indicador para a avaliação dos serviços de saúde ${ }^{1,2}$. A incorporação da satisfação do usuátio na avaliação dos serviços tem sido valorizada não apenas por constituir-se em indicador de qualidade, mas também por estar potencialmente relacionada à melhora na adesão ao tratamento, à relação médico-paciente e à maior adequação no uso do serviço ${ }^{3,4}$. A satisfação é estudada e pode ser definida em termos das expectativas e da percepção que os usuários têm dos servicos recebidos ${ }^{5}$. No Brasil, Kloetzel et al validaram um instrumento de mensuraçào da satisfação dos usuários a partir da experiència com relação à última consulta ${ }^{\circ}$. $\mathrm{O}$ referido estudo mostrou que a satisfação ê um indicador útil na orientação das medidas corretivas.

A Atenção Primária à Saúde (APS) é definida por meio de seus atributos, quais sejam, o acesso de primeiro contato ao Sistema de Saúde, a longitudinalidade, a integralidade da atenção, a coordenação da assistência, a orientação familiar e comunitária e a competência cultural'. Nesse sentico, os atributos da APS são reconhecidos na literatura internacional como eixos estruturantes do processo de atenção, associados à qualidade dos serviços", à efetividade" e à eficiência ${ }^{10}$ de suas intervenções. Starfield et al desenvolveram um instrumento capaz de medir sua presença e extensão, o Primary Care Assessment Tool (PCATool) ${ }^{\text {th }}$. Esse instrumento produz escores dos atributos e um escore geral sobre a qualidade do processo de atenção em APS. Assim, o uso de um instrumento capaz de avaliar a qualidade da atenção primária oferecida, a partir da mensuração da extensão dos atributos da APS, representa uma oportunidade de avaliação comparativa do grau de êxito dos serviços em alcançar excelência na prática de APS.

No Brasil, a Estratégia Saúde da Familia (ESE) apresenta-se como o modelo de APS orientador da reorganização dos serviços e do Sistema Único de Saúde (SLS) ${ }^{12}$. Existe, porém, heterogeneidade na qualidade assistencial das Equipes de Saúde da Família. Além disso, em muitos municípios, coexistem, juntamente com a ESF, outros modelos de atenção primária. Torna-se fundamental, portanto, a pesquisa em APS a partir da perspectiva do usuário, tanto para a avaliação da satisfação dele com relação aos serviços de APS, como também para a avaliação da qualidade do processo assistencial ${ }^{13}$.

Com esse desafio, o Grupo de Pesquisa em Atençao Primária à Saúde de Porto Alegre (GP.APS) desenvolveu uma pesquisa de base populacional para avaliar os servicos de atenção primária de Porto Alegre: a ESE, as Unidades Básicas de Saúde Tradicionais (UBS), o Serviço de Saúde Comunitária do Grupo Hospitalar Conceição (SSC/GHC), o Centro de Saúde-Escola Murialdo (CSEM) e a Caixa de 
Assistência dos Funcionários do Banco do Brasil (CASSIRS). Um dos objetivos da referida pesquisa, apresentado neste artigo, é comparar a satisfação dos usuários com os serviços de saúde entre os que avaliam a qualidade do processo de atenção recebida como sendo de alto escore de APS e os usuários que a avaliam como sendo de baixo escore.

\section{Metodologia}

Trata-se de um estudo transversal de base populacional realizado na cidade de Porto Alegre, RS. A populaçâo alvo do estudo foi adultos maiores de 18 anos de idade, residentes nas áreas geográficas cobertas pela rede de APS em Porto Alegre, composta por quatro serviços (ESF, UBS, SSC/GHC e CSEM) e a listagem de pacientes maiores de 18 anos atendidos pelas Equipes de Saúde da Família da CASSI-RS. A amostragem dos domicilios visitados foi por conglomerado (clusters), sendo estratificada por gerência distrital de saúde. Os critérios de inclusão foram: adultos maiores de 18 anos, residentes nos domicílios selecionados, que aceitarem livremente participar da pesquisa, após leitura, aceitação e assinatura do Termo de Consentimento Livre e Esclarecido. Os critérios de exclusāo foram: indivíduos maiores de 18 anos dos domicilios selecionados que não apresentarem condições de saúde para responder ao questionário, última consulta fora de Porto Alegre ou última consulta em Porto Alegre anterior a 1996.

A amostragem foi realizada por conglomerado de serviços e proporcional à população adscrita. $\mathrm{O}$ cálculo amostral foi feito com um poder de $80 \%$, nível de significância (a) de 5\%. A proporção de alto escore de APS entre os serviços foi estimada para variar entre $15 \%$ e $50 \%$.

Foi utilizado o Instrumento de Avaliação da Atenção Primária, PCATool-Brasil ${ }^{11}$. Esse instrumento mede a presença e a extensão dos quatro atributos essenciais (acesso de primeiro contato, longitudinalidade, integralidade e coordenação) e de dois atributos derivados (orientação familiar e orientação comunitária) da APS e o grau de afiliação do usuário ao serviço de saúde. Possibilita, por meio de respostas tipo Likert, construir escores de APS com inter- valo de 1-4 para cada atributo. O escore final de cada um desses atributos é dado pela média das respostas de seus itens (perguntas). Alguns são formados por subdimensões, como acesso (primeiro contato [A1] e utilização [A2]) e integralidade (serviços recebidos [11] e serviços disponiveis [12]). A média dos escores de todos os atributos e suas subdimensões produz o escore geral de APS (EG).

Para o cálculo do EG, a fórmula é:

\section{$\mathrm{EG}=\mathrm{GA}+\mathrm{A} 1+\mathrm{A} 2+\mathrm{L}+\mathrm{C}+\mathrm{I} 1+\mathrm{I} 2+\mathrm{OF}+\mathrm{OC}$}

\section{9}

São considerados serviços com alto escore de APS aqueles que obtiverem valor do escore geral maior ou igual a 6,6 .

A satisfação dos usuários foi medida por um questionário (Quadro 1) validado no Brasil ${ }^{6}$, composto de 12 questões referentes a distintos aspectos relacionados à última consulta. Esse instrumento é constituído de respostas tipo Likert de 1-5, representadas por figuras de faces com cinco expressões distintas de satisfação ("muito bom - $A$ ", "bom - B", "regular - C", "ruim - D" e "muito ruim - E"), conforme Figura 1. Os entrevistados eram convidados a responder às perguntas baseando-se na sua experiência da última consulta na rede de atenção primária de Porto Alegre nos 12 meses anteriores à entrevista. Os aspectos avaliados foram: facilidade de acesso à consulta, tempo dispendido na sala de espera, cordialidade por parte da recepção, cordialidade do profissional, atenção dada às queixas, impressão do exame clínico, confiança despertada pelo profissional, confiança na receita, explicações do médico com respeito à doença, explicações quanto ao prognóstico, satisfação com o agendamento e avaliação geral da consulta.

Para a análise, os resultados foram agrupados em duas classificações, sendo "satisfeito" quando referido "muito bom" ou "bom", e "insatisfeito" quando referido algum dos demais itens. Um teste qui-quadrado de homogeneidade foi empregado para a comparação entre a proporçào de profissionais satisfeitos que atribuíram baixo e alto escore aos serviços de atenção primária de Porto Alegre. Foi adotado o nível de significância de 5\% para a análise estatística. As análises foram realizadas utilizando os 
softwares SPSS, versão 13.0, e Stata, versão 9.0.

Maiores detalhes sobre a metodologia do estudo podem ser consultados na publicação de seu protocolo de pesquisa, intitulado "Quality and effectiveness of different approaches to primary care delivery in Brazil"14, disponivel no endereço http:/ / www.biomedcentral.com/1472-6963/ 6/156.

O projeto principal, intitulado "Avaliação da qualidade do processo de atenção e da sua efetividade sobre a saúde do adulto no Programa Saúde da Família e em mode- los alternativos no município de Porto Alegre", tem aprovação dos comitês de ética das instituições envolvidas na pesquisa: Secretaria Municipal de Saúde de Porto Alegre (SMS), Centro de Saúde Escola Murialdo (CSEM), Grupo Hospitalar Conceição (GHC), Hospital de Clínicas de Porto Alegre (HCPA), Caixa de Assistência dos Funcionários do Banco do Brasil - Regional RS (CASSI-RS) e Universidade Federal do Rio Grande do Sul (UFRGS). As entrevistas somente foram realizadas com os adultos que leram, concordaram e assinaram o Termo de Consentimento Livre e Esclarecido.

\section{Quadro 1.}

\section{Instrumento de avaliação da satisfação dos usuários}

Qual é a sua opinião sobre a facilidade de acesso a esta consulta (foi fácil marcar ou conseguir esta consulta)?

Qual é a sua opinião sobre o tempo dispendido na sala de espera (o que você achou do tempo que teve de esperar para consultar)?

Qual é a sua opinião sobre a cordialidade por parte da recepção (o pessoal da recepção foi cordial, educado, simpatico)?

Qual é a sua opiniào sobre a cordialidade do profissional que the atendeu (o médico/enfermeira foi cordial, educado(a), simpático(a))?

Qual é a sua opinião sobre a atenção dada as suas queixas pelo profissional que the atendeu (o médico/enfermeira ouviu com atenção o motivo da consulta, suas queixas)?

Qual é a sua opinião sobre o exame clínico (qual sua opinião sobre o jeito como o médico/enfermeira lhe examinou)?

Qual é a sua opiniào sobre a confiança despertada pelo médico/enfermeira (você confiou no médico/ enfermeira)?

Qual é a sua opinião sobre a confiança na receita (vocé confiou na receita dada pelo médico/enfermeira)?

Qual é a sua opiniào sobre as explicaçōes com respeito a sua doença (explicaçôes do médico/enfermeira sobre o motivo da consulta, sobre o seu problema, que o médico/enfermeira lhe deu na consulta)?

Qual é a sua opinião sobre as explicações quanto ao prognóstico (explicações do médico/enfermeira sobre a duração, a evolução e as consequencias do seu problema doença)?

Você ficou satisfeito com o agendamento (com a forma de marcação desta consulta)?

Qual é a sua avaliação geral desta consulta (opinião geral sobre a consulta como um todo)? 
Figura 1.

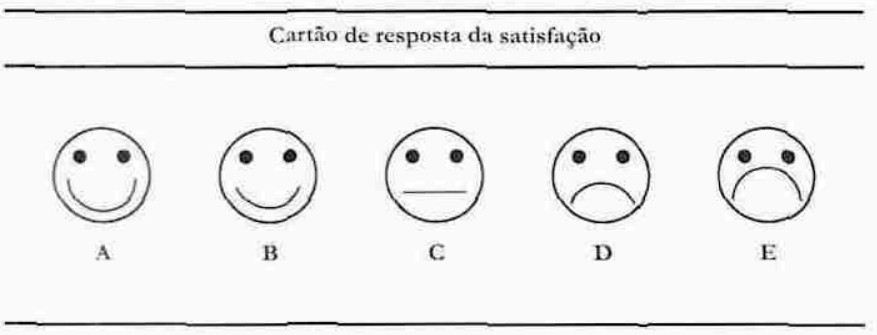

\section{Resultados}

O PCATool-Brasil foi aplicado a 1.393 usuários da rede de atenção primária de Porto Alegre. Desses, 1.154 realizaram a sua última consulta no periodo de 12 meses anteriores à entrevista, respondendo, assim, ao questionário de satisfação. $\mathrm{Na}$ amostra de $1.154,389$ (33,7\%) eram do sexo masculino e 765 ( $66,3 \%)$, do sexo feminino, sendo a idade média de 45,7 anos (desvio padrão=16,7). Dos entrevistados, $747(64,7 \%)$ classificaram a cor da pele como branca. Com relação à classe socioeconômica, $52,4 \%$ dos respondentes encontram-se na classe $\mathrm{C}$ e a renda per capita apresentou o valor médio de R\$ 750,5 (desvio padrão=
R\$1.146,4). A Tabela 1 apresenta a caracterização da amostra de 1.154 usuários.

A Tabela 2 apresenta o grau de satisfação dos usuários entre os serviços classificados como de alto ou baixo escore de APS. Observou-se diferença significativa nas 12 variáveis que refletem a satisfação, demonstrando sempre maior satisfação nos usuários que classificaram o seu serviço como de alto escore de APS $(\mathrm{p}<0,001)$. Embora todos os aspectos tenham sido bem avaliados nos serviços de alto escore, destacam-se os aspectos relacionados à relação médico-pessoa. Os itens com maior grau de satisfação foram a cordialidade do profissional, a atenção dada às queixas, a confiança despertada pelo profissional, a confiança na receita e as explicações com respeito à doença. Já os aspectos relacionados ao funcionamento do serviço de APS, como o tempo dispendido na sala de espera, a cordialidade por parte da recepção e a satisfação com o agendamento, apresentaram os percentuais mais baixos de grau de satisfação, tanto nos serviços de baixo como nos de alto escore da APS.

\section{Tabela 1.}

Distribuição socioeconômica da população vinculada aos serviços de APS, Porto Alegre, 2007.

\begin{tabular}{lc}
\hline Variáveis sociodemográficas & Média (desvio padrão) ou \% $\mathrm{n}=1154$ \\
Idade (anos) & $45,7(16,7)$ \\
Sexo & Masculino \\
Feminino & 33,7 \\
Escolaridade (anos completos) & 66,3 \\
Cor da pele & $8,3(4,6)$ \\
Brancos & \\
Não Brancos & 64,7 \\
Classe Econômica (Critério Brasil, 2007) & 35,1 \\
A & 3,5 \\
C & 28,5 \\
D & 52,4 \\
E & 13,8 \\
Renda Per Capita (reais, RS) & 1,8 \\
\hline
\end{tabular}


Tabela 2.

Comparação da proporção de profissionais satisfeitos entre os que atribuiram baixo ou alto escore aos serviços de atenção primária de Porto Alegre, 2007.

\begin{tabular}{|c|c|c|c|}
\hline & Baixo Escore & Alto Escore & \\
\hline Variáveis & $\begin{array}{c}\% \text { de usuários } \\
\text { satisfeitos (Ic95\%) }\end{array}$ & $\begin{array}{c}\% \text { de usuários } \\
\text { satisfeitos (Ic95\%) }\end{array}$ & valor-p \\
\hline Facilidade de acesso à consulta & $71,6(68,5-74,5)$ & $90,9(86,8-94,0)$ & $<0.001$ \\
\hline Tempo dispendido na sala de espera & $52,4(49,0-55,7)$ & $78,5(73,1-83,2)$ & $<0.001$ \\
\hline Cordialidade por parte da recepção & $72,7(69,6-75,6)$ & $86,5(81,8-90,3)$ & $<0.001$ \\
\hline Cordialidade do profissional & $87,3(84,9-89,4)$ & $98,5(96,3-99,6)$ & $<0.001$ \\
\hline Atenção dada às queixas & $81,2(78,5-83,7)$ & $95,3(92,0-97,4)$ & $<0.001$ \\
\hline Impressão do exame clínico & $77,2(74,3-79,9)$ & $94,5(91,1-96,9)$ & $<0.001$ \\
\hline Confiança despertada pelo profissional & $76,7(73,7-79,4)$ & $97,4(94,8-98,9)$ & $<0.001$ \\
\hline Confiança na receita & $81,5(78,8-84,0)$ & $95,6(92,4-97,7)$ & $<0.001$ \\
\hline Explicaçōes do médico com respeito à doença & $76,3(73,4-79,1)$ & $96,0(92,9-97,9)$ & $<0.001$ \\
\hline Explicações quanto ao prognóstico & $71,5(68,4-74,5)$ & $93,4(89,8-96,0)$ & $<0.001$ \\
\hline Satisfaça com o agendamento & $70,1(66,9-73,1)$ & $85,8(81,1-89,7)$ & $<0.001$ \\
\hline Avaliação geral da consulta & $73,5(70,4-76,4)$ & $95,6(92,5-97,7)$ & $<0.001$ \\
\hline
\end{tabular}

\section{Discussão}

Os resultados apresentados assemelham-se aos encontrados no estudo de validação do instrumento de satisfação ${ }^{6}$, o qual mostrou menor satisfação com os aspectos referentes ao funcionamento do serviço, ao acesso e ao tempo dispendido na sala de espera, e maior satisfação com os aspectos referentes à relação médico-pessoa. Dessa forma, destacamos a importância da atuação do profissional de APS para a produção de melhores resultados em satisfação com os serviços de saúde.

Os serviços classificados pelos usuários como de alto escore apresentaram um grau maior de satisfação em todos os aspectos avaliados quando comparados aos serviços de baixo escore de APS. Os altos percentuais de satisfação verificados concordam com o efeito descrito na literatura como de "elevação da satisfação" nos estudos sobre o tema ${ }^{5}$. Entretanto, nesse estudo, diferenciamos dois grupos de satisfação distintos: o dos usuários que percebem a qualidade do processo de atenção recebida como sendo de alto escore, e o dos usuários que a percebem como sendo de baixo escore. Dessa forma, encontramos associação entre o grau de satisfação e a qualidade do processo de atenção percebida.

No tratamento estatístico dos dados, foi realizada uma análise univariada, que, se por um lado possibilitou a identificação da associação colocada acima, por outro apresenta suas limitações. Para fazer uma avaliaçào mais precisa do peso de cada varíavel na produção da associação encontrada e também para considerar a influência de outras variáveis no desfecho satisfação, uma análise multivariada pode ser realizada. Além disso, por se tratar de um estudo transversal, os resultados não estão livres de causalidade reversa. Por exemplo, não podemos afirmar se a percepção de alto escore feita pelo usuário é a razão da maior satisfação com o serviço ou se a maior satisfação é o motivo da atribuição de um alto escore.

\section{Considerações finais}

Considera-se que a satisfação é um desfecho em saúde que pode ser facilmente mensurado. A sua medida orienta o planejamento e as intervenções para a qualificação do serviço. Com os dados apresentados, verificamos, por exemplo, que medidas relativas à melhoria do acesso devem ser tomadas. Para a identificação, porém, de quais serviços devem prioritariamente fazer essa adequação, é necessária uma análise da satisfação por tipo de serviço, ou seja, modelo de atenção.

Como a maior satisfação foi relacionada aos aspectos refe- 
rentes à relação médico-pessoa, pode-se também colocála como um indicador da qualificação do profissional de APS. Estudos posteriores são necessários para investigar quais os aspectos da qualificação profissional se relacionam com a satisfação dos usuários na consulta. Por exemplo, para investigar o efeito da especialidade médica Medicina de Família e Comunidade comparado com o efeito das demais especialidades na satisfação dos usuários com a consulta em APS. Por fim, com base nos resultados apresentados, pode-se considerar que os serviços devem ser orientados pelos atributos da APS para atingirem altos níveis de satisfação dos usuários.

\section{Conflitos de interesse}

Os autores declaram não existir conflitos de interesse.

\section{Instituições financiadoras}

Coordenação de Aperfeiçoamento de Pessoal de Nivel Superior do Ministério da Educação (CAPES), Conselho Nacional de Desenvolvimento Científico e Tecnológico $(\mathrm{CNPq})$ e Fundação de Amparo à Pesquisa do Estado do Rio Grande do Sul (Fapergs).

\section{Referências}

1. Starfield B. Measurement of Outcome: A Ptoposed Scheme. The Milbank Quarterly. 2005; 83 (4): 1-11.

2. Sans-Corrales $M$ et al. Family medicine attributes related to satisfaction, health and costs. Family Practice. 2006; 23 (3): $308-316$.

3. Trad L, Bastos AC, Santana EM, Nunes M. A construçào social da estratégia de saúde da família: condições, sujeitos e contextos. [s.1.]: PNEPG; 2001.

4. Howard $\mathrm{M}$ et al. Patient satisfaction with care for urgent health problems: a survey of family practice patients. Ann Fam Med. 2007; (5): 419-424.

5. Esperidião M, Trad L. Avaliação de satisfação de usuários. Ciência \& Saúde Coletiva. 2005; 10 (supl.): 303-312. 6. Kloetzel $\mathrm{K}$ et al. Controle de qualidade em atenção primária à saúdẻ a satisfação do usuário. Cad. Saúde Pública.
1998; 14(3): 623-628.

7. Starfield B. Atenção primária: equilibrio entre necessidades de saúde, serviços e tecnologia. Brasília (DF): Unesco, Ministério da Saúde; 2004.

8. Marques RM, Mendes A. Atenção Básica e Programa de Saúde da Família (PSF): novos rumos para a política de saúde e seu financiamento? Ciência Saúde Coletiva. 2003; 8(2): 403-15.

9. Bodstein R. Atenção básica na agenda da saúde. Ciência Saúde Coletiva. 2002; 7(3): 401-12.

10. Shi L, Starfield B, Politzer R, Regan J. Primary care, selfrated health, and reductions in social disparities in health. Health Serv Res. 2002; 37(3): 529-550.

11. Shi L, Starfield B, Jihaong X. Validating the Adult Primary Care Assessment Tool. J Fam Practice. 2001; 50(2): 161175.

12. Brasil. Ministério da Saúde. Política Nacional de Atenção Básica. Portaria 648; 2006.

13. Harzheim E. Evaluación de la atención a la salud infantil del Programa Saúde da Familia en la región sur de Porto Alegre, Brasil. [Tese]. Alicante: Departamento de Salud Pública, Universidad de Alicante; 2004.

14. Harzheim E et al. Quality and effectiveness of different approaches to primary care delivery in Brazil. BMC Health Services Research. 2006; 6: 156.

\section{Endereço para correspondência:}

Rua Carlos Silveira Martins Pacheco, 55, Bloco F, Apt 901 , Cristo Redentor

Porto Alegre RS - CEP: 91.350-300

\section{Endereço eletrônico:}

rocaprio@pro.via-rs.com.br 